\title{
Ascent sequences and upper triangular matrices containing non-negative integers
}

\author{
Mark Dukes* \\ Mathematics Division \\ Science Institute, \\ University of Iceland, \\ 107 Reykjavík, Iceland \\ dukes@hi.is
}

\author{
Robert Parviainen \\ The Mathematics Institute, \\ School of Computer Science, \\ Reykjavík University, \\ 103 Reykjavík, Iceland \\ parviainen@ru.is
}

Submitted: Jan 25, 2010; Accepted: Mar 22, 2010; Published: Mar 29, 2010

Mathematics Subject Classifications: 05A05, 05A19

\begin{abstract}
This paper presents a bijection between ascent sequences and upper triangular matrices whose non-negative entries are such that all rows and columns contain at least one non-zero entry. We show the equivalence of several natural statistics on these structures under this bijection and prove that some of these statistics are equidistributed. Several special classes of matrices are shown to have simple formulations in terms of ascent sequences. Binary matrices are shown to correspond to ascent sequences with no two adjacent entries the same. Bidiagonal matrices are shown to be related to order-consecutive set partitions and a simple condition on the ascent sequences generate this class.
\end{abstract}

\section{Introduction}

Let $\operatorname{lnt}_{n}$ be the collection of upper triangular matrices with non-negative integer entries which sum to $n \in \mathbb{N}$ such that all rows and columns contain at least one non-zero entry. For example,

$$
\operatorname{lnt}_{3}=\left\{(3),\left(\begin{array}{ll}
2 & 0 \\
0 & 1
\end{array}\right),\left(\begin{array}{ll}
1 & 1 \\
0 & 1
\end{array}\right),\left(\begin{array}{ll}
1 & 0 \\
0 & 2
\end{array}\right),\left(\begin{array}{lll}
1 & 0 & 0 \\
0 & 1 & 0 \\
0 & 0 & 1
\end{array}\right)\right\} .
$$

\footnotetext{
${ }^{*}$ Both authors were supported by grant no. 090038011 from the Icelandic Research Fund.
} 
We use the standard notation $[a, b]$ for the interval of integers $\{a, a+1, \ldots, b\}$ and define $[n]=[1, n]$. Given a sequence of integers $y=\left(y_{1}, \ldots, y_{n}\right)$, we say that $y$ has an ascent at position $i$ if $y_{i}<y_{i+1}$. The number of ascents of $y$ is denoted by $\operatorname{asc}(y)$. Let $\mathcal{A}_{n}$ be the collection of ascent sequences of length $n$ :

$$
\mathcal{A}_{n}=\left\{\left(x_{1}, \ldots, x_{n}\right): x_{i} \in\left[0,1+\operatorname{asc}\left(x_{1}, \ldots, x_{i-1}\right)\right], \text { for all } 1<i \leqslant n\right\},
$$

where $x_{1}:=0$ and $\operatorname{asc}\left(x_{1}\right):=0$. For example,

$$
\mathcal{A}_{3}=\{(0,0,0),(0,0,1),(0,1,0),(0,1,1),(0,1,2)\}
$$

These sequences were introduced in the recent paper by Bousquet-Mélou et al. [1] and were shown to unify three combinatorial structures: $(\mathbf{2}+\mathbf{2})$-free posets, a class of pattern avoiding permutations and a class of involutions that are sometimes termed chord diagrams. This paper complements the results of [1] by presenting a fourth structure, the matrices in $\operatorname{lnt}_{n}$, that can be encoded by an ascent sequence of length $n$. To this end we have attempted to use notation that is indicative of the transformations and operations in the original paper [1]. The bijection presented in this paper is used in Dukes et al. [2] to resolve a conjecture concerning the number of binary matrices in $\operatorname{lnt}_{n}$, and presents a generating function for the number of matrices whose entries are bounded by some value $k$.

The class of matrices we study here have been touched upon in the literature before. The binary case is known to encode a subclass of interval orders (the full class of interval orders are in bijection with $(\mathbf{2}+\mathbf{2})$-free posets), see Fishburn [3]. Mitas [5] used our class of matrices to study the jump number problem on interval orders, but without a formal statement or proof of any bijection, and without studying further properties of the relation.

In section 2 we present a bijection $\Gamma$ from matrices in $\operatorname{Int}_{n}$ to ascent sequences in $\mathcal{A}_{n}$. In section 3 we show how statistics on both of these structures are related under $\Gamma$ and prove that some of the statistics are equidistributed. Section 4 looks at properties of restricted sets of matrices and ascent sequences which give rise to interesting structures, order-consecutive set partitions being one example. We end with some open problems in section 5 .

\section{Upper triangular matrices}

In this section we will define a removal and an addition operation on matrices in Int $_{n}$ that are essential for the bijection. These operations have the effect of decreasing (resp. increasing) the sum of the entries in a matrix by 1.

Given $A \in \operatorname{lnt}_{n}$ let $\operatorname{dim}(A)$ be the number of rows in the matrix $A$. Furthermore, let index $(A)$ be the smallest value of $i$ such that $A_{i, \operatorname{dim}(A)}>0$ and define value $(A):=$ 
$A_{\text {index }(A), \operatorname{dim}(A)}$. Let $\operatorname{rowsum}_{i}(A)$ and $\operatorname{colsum}_{i}(A)$ be the sum of the elements in row $i$ and column $i$ of $A$, respectively.

Consider the following operation $f$ on a given matrix $A \in \operatorname{Int}_{n}$.

(Rem1) If $\operatorname{rowsum}_{\operatorname{index}(A)}(A)>1$ then let $f(A)$ be the matrix $A$ with the entry $A_{\text {index }(A), \operatorname{dim}(A)}$ reduced by 1 .

(Rem2) If $\operatorname{rowsum}_{\operatorname{index}(A)}(A)=1$ and $\operatorname{index}(A)=\operatorname{dim}(A)$, then let $f(A)$ be the matrix $A$ with row $\operatorname{dim}(A)$ and column $\operatorname{dim}(A)$ removed.

(Rem3) If $\operatorname{rowsum}_{\operatorname{index}(A)}(A)=1$ and $\operatorname{index}(A)<\operatorname{dim}(A)$, then we form $f(A)$ in the following way. Let $A_{i, \operatorname{dim}(A)}=A_{i, \operatorname{index}(A)}$ for all $1 \leqslant i \leqslant \operatorname{index}(A)-1$. Now simultaneously delete row index $(A)$ and column index $(A)$. Let the resulting $(\operatorname{dim}(A)-1) \times(\operatorname{dim}(A)-1)$ matrix be $f(A)$.

Example 1. Consider the following three matrices:

$$
A=\left(\begin{array}{cccc}
1 & 0 & 1 & 0 \\
0 & 2 & 0 & 3 \\
0 & 0 & 1 & 4 \\
0 & 0 & 0 & 2
\end{array}\right) ; \quad B=\left(\begin{array}{cccc}
5 & 1 & 3 & 0 \\
0 & 1 & 0 & 0 \\
0 & 0 & 1 & 0 \\
0 & 0 & 0 & 1
\end{array}\right) ; \quad C=\left(\begin{array}{ccccccc}
1 & 0 & 0 & 1 & 0 & 0 & 0 \\
0 & 1 & 0 & 1 & 1 & 0 & 0 \\
0 & 0 & 1 & 2 & 1 & 1 & 0 \\
0 & 0 & 0 & 0 & 0 & 0 & 1 \\
0 & 0 & 0 & 0 & 0 & 1 & 0 \\
0 & 0 & 0 & 0 & 0 & 0 & 1 \\
0 & 0 & 0 & 0 & 0 & 0 & 1
\end{array}\right) .
$$

For matrix $A$, rule $\operatorname{Rem} 1$ applies since value $(A)=3$ and

$$
f(A)=\left(\begin{array}{llll}
1 & 0 & 1 & 0 \\
0 & 2 & 0 & 2 \\
0 & 0 & 1 & 4 \\
0 & 0 & 0 & 2
\end{array}\right)
$$

For matrix $B$, since value $(B)=1$ and $\operatorname{index}(B)=\operatorname{dim}(B)=4$ rule Rem2 applies and

$$
f(B)=\left(\begin{array}{lll}
5 & 1 & 3 \\
0 & 1 & 0 \\
0 & 0 & 1
\end{array}\right)
$$

For matrix $C$, since value $(C)=1,4=\operatorname{index}(C)<\operatorname{dim}(C)=7$, and all other entries in row index $(C)=4$ are zero, then we form $f(C)$ in the following way: first copy the index $(C)-1=3$ highest entries in column index $(C)$ to the top index $(C)-1=3$ entries in column $\operatorname{dim}(C)=7$. These are illustrated in bold in the following matrix:

$$
\left(\begin{array}{lllllll}
1 & 0 & 0 & 1 & 0 & 0 & \mathbf{1} \\
0 & 1 & 0 & 1 & 1 & 0 & \mathbf{1} \\
0 & 0 & 1 & 2 & 1 & 1 & \mathbf{2} \\
0 & 0 & 0 & 0 & 0 & 0 & 1 \\
0 & 0 & 0 & 0 & 0 & 1 & 0 \\
0 & 0 & 0 & 0 & 0 & 0 & 1 \\
0 & 0 & 0 & 0 & 0 & 0 & 1
\end{array}\right)
$$


Next we simultaneously remove column index $(C)=4$ and row index $(C)=4$ to get $f(C)$ :

$$
\left(\begin{array}{llllll}
1 & 0 & 0 & 0 & 0 & \mathbf{1} \\
0 & 1 & 0 & 1 & 0 & \mathbf{1} \\
0 & 0 & 1 & 1 & 1 & \mathbf{2} \\
& & & & \\
0 & 0 & 0 & 0 & 1 & 0 \\
0 & 0 & 0 & 0 & 0 & 1 \\
0 & 0 & 0 & 0 & 0 & 1
\end{array}\right) \Longrightarrow f(C)=\left(\begin{array}{llllll}
1 & 0 & 0 & 0 & 0 & \mathbf{1} \\
0 & 1 & 0 & 1 & 0 & \mathbf{1} \\
0 & 0 & 1 & 1 & 1 & \mathbf{2} \\
0 & 0 & 0 & 0 & 1 & 0 \\
0 & 0 & 0 & 0 & 0 & 1 \\
0 & 0 & 0 & 0 & 0 & 1
\end{array}\right) .
$$

We now show that the above removal operation yields an upper triangular matrix in $\operatorname{Int}_{n-1}$. If $\operatorname{index}(A)=i+1$ and the above removal operation, applied to $A$, gives $f(A)$, then we define $\psi(A)=(f(A), i)$. Notice that $1 \leqslant \operatorname{index}(A) \leqslant \operatorname{dim}(A)$.

Lemma 1. If $n \geqslant 2, A \in \operatorname{Int}_{n}$ and $\psi(A)=(B, i)$, then $B \in \operatorname{Int}_{n-1}$.

Proof. It is easy to see that the sum of the entries in $B$ is one less than the sum of the entries in $A$. It remains to show that there are no columns or rows of zeros in $B$. This is trivial to see for the removal operations Rem1 and Rem2. For rule Rem3, it is clear that $\operatorname{rowsum}_{i}(B)=\operatorname{rowsum}_{i}(A)>0$ and $\operatorname{colsum}_{i}(B)=\operatorname{colsum}_{i}(A)>0$ for all $1 \leqslant i<\operatorname{index}(A)$. Also we have $\operatorname{rowsum}_{i}(B)=\operatorname{rowsum}_{i+1}(A)>0$ for all index $(A) \leqslant i \leqslant$ $\operatorname{dim}(A)-1$ and $\operatorname{colsum}_{i}(B)=\operatorname{colsum}_{i+1}(A)>0$ for all index $(A) \leqslant i<\operatorname{dim}(A)-1$. Finally colsum $_{\operatorname{dim}(A)-1}(B)=$ colsum $_{\text {index }(A)}(A)+$ colsum $_{\operatorname{dim}(A)-1}(A)-1>0$.

We now define the complementary addition rules for each of the removal steps. Their consistency will be shown later. Given $A \in \operatorname{Int}_{n}$ and $m \in[0, \operatorname{dim}(A)]$ we construct the matrix $\phi(A, m)$ in the following manner.

(Add1) If $0 \leqslant m \leqslant \operatorname{index}(A)-1$ then let $\phi(A, m)$ be the matrix $A$ with the entry at position $(m+1, \operatorname{dim}(A))$ increased by 1 .

(Add2) If $m=\operatorname{dim}(A)$ then let $\phi(A, m)$ be the matrix $\left(\begin{array}{cc}A & 0 \\ 0 & 1\end{array}\right)$.

(Add3) If index $(A) \leqslant m<\operatorname{dim}(A)$ then form $\phi(A, m)$ in the following way:

In $A$, insert a new (empty) row between rows $m$ and $m+1$, and insert a new (empty) column between columns $m$ and $m+1$. Let the new row be filled with all zeros except for the rightmost entry which is 1 . Move each of the entries above this new rightmost one to the new column between columns $m$ and $m+1$ and replace them with zeros. Finally let all other entries in the new column be zero. The resulting matrix is $\phi(A, m)$.

Example 2. Consider the following three matrices:

$$
A=\left(\begin{array}{cccc}
1 & 0 & 1 & 0 \\
0 & 2 & 0 & 0 \\
0 & 0 & 1 & 5 \\
0 & 0 & 0 & 1
\end{array}\right) ; \quad B=\left(\begin{array}{cccc}
1 & 5 & 0 & 4 \\
0 & 1 & 0 & 3 \\
0 & 0 & 1 & 2 \\
0 & 0 & 0 & 3
\end{array}\right) ; \quad C=\left(\begin{array}{cccccc}
1 & 0 & 0 & 0 & 6 & 0 \\
0 & 1 & 0 & 1 & 0 & 7 \\
0 & 0 & 1 & 1 & 1 & 2 \\
0 & 0 & 0 & 0 & 3 & 0 \\
0 & 0 & 0 & 0 & 0 & 1 \\
0 & 0 & 0 & 0 & 0 & 1
\end{array}\right)
$$


In order to form $\phi(A, 1)$, since $m=1 \leqslant \operatorname{index}(A)-1=2$ we see that rule Add1 applies and

$$
\phi(A, 1)=\left(\begin{array}{llll}
1 & 0 & 1 & 0 \\
0 & 2 & 0 & 1 \\
0 & 0 & 1 & 5 \\
0 & 0 & 0 & 1
\end{array}\right) .
$$

In order to form $\phi(B, 4)$, since $m=4=\operatorname{dim}(B)$ we see that rule Add2 applies and

$$
\phi(B, 4)=\left(\begin{array}{lllll}
1 & 5 & 0 & 4 & 0 \\
0 & 1 & 0 & 3 & 0 \\
0 & 0 & 1 & 2 & 0 \\
0 & 0 & 0 & 3 & 0 \\
0 & 0 & 0 & 0 & 1
\end{array}\right)
$$

In order to form $\phi(C, 3)$, since index $(C)=2 \leqslant 3<5=\operatorname{dim}(C)$ we see that rule Add3 applies and we do as follows. Insert a new empty row and column between rows 3 and 4 and columns 3 and 4 of $C$ :

$$
\left(\begin{array}{llllll}
1 & 0 & 0 & 0 & 6 & 0 \\
0 & 1 & 0 & 1 & 0 & 7 \\
0 & 0 & 1 & 1 & 1 & 2 \\
0 & 0 & 0 & 0 & 3 & 0 \\
0 & 0 & 0 & 0 & 0 & 1 \\
0 & 0 & 0 & 0 & 0 & 1
\end{array}\right)
$$

Fill the empty row with all zeros and a rightmost 1, this is highlighted in bold. Next move the entries above the new 1 to the new column and replace them with zeros.

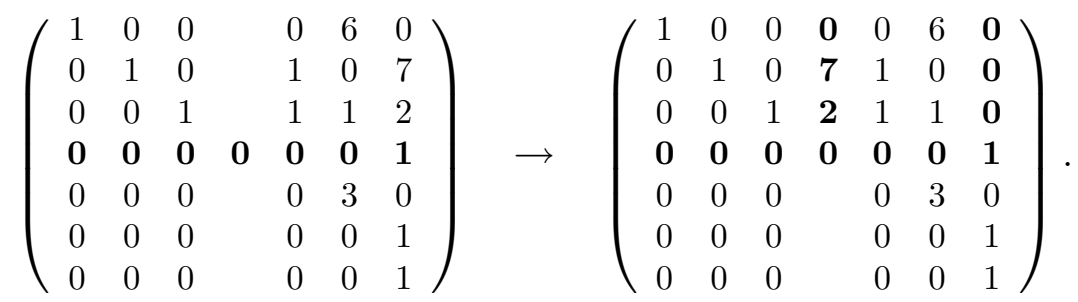

Finally fill the remaining empty positions with zeros to yield $\phi(C, 3)$ :

$$
\phi(C, 3)=\left(\begin{array}{lllllll}
1 & 0 & 0 & \mathbf{0} & 0 & 6 & \mathbf{0} \\
0 & 1 & 0 & \mathbf{7} & 1 & 0 & \mathbf{0} \\
0 & 0 & 1 & \mathbf{2} & 1 & 1 & \mathbf{0} \\
\mathbf{0} & \mathbf{0} & \mathbf{0} & \mathbf{0} & \mathbf{0} & \mathbf{0} & \mathbf{1} \\
0 & 0 & 0 & \mathbf{0} & 0 & 3 & 0 \\
0 & 0 & 0 & \mathbf{0} & 0 & 0 & 1 \\
0 & 0 & 0 & \mathbf{0} & 0 & 0 & 1
\end{array}\right)
$$

We now show that this addition operation yields another upper triangular matrix where every row and column contain at least one non-zero entry.

Lemma 2. If $n \geqslant 2, B \in \operatorname{Int}_{n-1}, 0 \leqslant i \leqslant \operatorname{dim}(B)$ and $A=\phi(B, i)$, then $A \in \operatorname{Int}_{n}$ and index $(A)=i+1$. 
Proof. In each of the operations, Add1, Add2 and Add3, the sum of the entries of the matrix is increased by exactly 1 . It is straightforward to check that each row and column contains at least one non-zero entry. The property of being upper-triangular is also preserved. Thus it is clear that $A=\phi(B, i) \in \operatorname{lnt}_{n}$.

It is similarly straightforward to check that index $(A)=i+1$ in each of the three cases.

Lemma 3. For any $B \in \operatorname{lnt}_{n}$ and integer $i$ such that $0 \leqslant i \leqslant \operatorname{dim}(B)$, we have $\psi(\phi(B, i))=$ $(B, i)$. If $n>1$ then we also have $\phi(\psi(B))=B$.

Proof. First let us denote $A=\phi(B, i)$. From Lemma 2 above index $(A)=i+1$ and so the removal operation when applied to $A$ will yield $\psi(A)=(C, i)$ for some matrix $C$. Thus we need only show that $B=C$ for each of the three cases.

Let us assume that $0 \leqslant i \leqslant \operatorname{index}(B)-1$. Then $A$ is simply a copy of $B$ with the entry at position $(i+1, \operatorname{dim}(B))$ increased by one. Similarly, rule Rem1 applies for $A$ and so $C$ will be the same as $A$ except that the entry at position $(\operatorname{index}(A), \operatorname{dim} B)=(i+1, \operatorname{dim} B)$ is decreased by one. Thus $B=C$.

Assume next that $i=\operatorname{dim}(B)$, so that rule Add2 applies and $A=\left(\begin{array}{cc}B & 0 \\ 0 & 1\end{array}\right)$. Since index $(A)=\operatorname{dim}(A)$, rule Rem2 applies and we remove both column and $\operatorname{row} \operatorname{dim} A$ of $A$ to get $C=(B)$.

If $\operatorname{index}(B) \leqslant i<\operatorname{dim}(B)$ then rule Add3 applies. For this, $B$ must have the following form

$$
B=\left(\begin{array}{ccc}
X & Y & e_{1} \\
\vdots \\
e_{i} \\
0 & Z & e_{i+1} \\
\vdots \\
e_{n}
\end{array}\right)
$$

where at least one of $\left\{e_{1}, \ldots, e_{i}\right\}$ is non-zero. From this we find that

$$
A=\left(\begin{array}{cccc}
X & e_{1} & Y & 0 \\
& \vdots & Y & \vdots \\
0 \cdots 0 & 0 & 0 \cdots 0 & 1 \\
0 & 0 & Z & e_{i+1} \\
& \vdots & & \vdots \\
& 0 & & e_{n}
\end{array}\right)
$$

Since index $(A)=i+1, \operatorname{value}(A)=1$ and all other entries in this row are zero, the removal 
operation to be applied is Rem3 and we find that

$$
C=\left(\begin{array}{ccc}
X & Y & e_{1} \\
& & e_{i} \\
& & e_{i+1} \\
0 & Z & \vdots \\
e_{n}
\end{array}\right)=B .
$$

The second statement follows by applying a similar analysis of the addition and removal operations.

We now define a map $\Gamma$ from $\operatorname{Int}_{n}$ to $\mathcal{A}_{n}$ recursively as follows. For $n=1$ we let $\Gamma((1))=$ (0). Now let $n \geqslant 2$ and suppose that the removal operation, when applied to $A \in \operatorname{Int}_{n}$, gives $\psi(A)=(B, i)$. Then the sequence associated with $A$ is $\Gamma(A):=\left(x_{1}, \ldots, x_{n-1}, i\right)$, where $\left(x_{1}, \ldots, x_{n-1}\right)=\Gamma(B)$. For example, $\Gamma$ maps the $i$ th element of $\operatorname{Int}_{3}$ to the $i$ th element of $\mathcal{A}_{3}$ as they are listed in the introduction.

Theorem 4. The map $\Gamma: \operatorname{lnt}_{n} \mapsto \mathcal{A}_{n}$ is a bijection.

Proof. Since the sequence $\Gamma(A)$ encodes the construction of the matrix $A$, the map $\Gamma$ is injective. We want to prove that the image of $\operatorname{lnt}_{n}$ is the set $\mathcal{A}_{n}$. The recursive description of the map $\Gamma$ tells us that $x=\left(x_{1}, \ldots, x_{n}\right) \in \Gamma\left(\operatorname{Int}_{n}\right)$ if and only if

$$
x^{\prime}=\left(x_{1}, \ldots, x_{n-1}\right) \in \Gamma\left(\operatorname{lnt}_{n-1}\right) \quad \text { and } \quad 0 \leqslant x_{n} \leqslant \operatorname{dim}\left(\Gamma^{-1}\left(x^{\prime}\right)\right) .
$$

We will prove by induction on $n$ that for all $A \in \operatorname{Int}_{n}$, with associated sequence $\Gamma(A)=$ $x=\left(x_{1}, \ldots, x_{n}\right)$, one has

$$
\operatorname{dim}(A)=\operatorname{asc}(x) \quad \text { and } \quad \operatorname{index}(A)=x_{n}+1 .
$$

Clearly, this will convert the above description (1) of $\Gamma(A)$ into the definition of ascent sequences, thus concluding the proof.

So let us focus on the properties (2). They hold for $n=1$. Assume they hold for some $n-1$ with $n \geqslant 2$, and let $A=\phi(B, i)$ for $B \in \operatorname{Int}_{n-1}$. If $\Gamma(B)=x^{\prime}=\left(x_{1}, \ldots, x_{n-1}\right)$ then $\Gamma(A)=x=\left(x_{1}, \ldots, x_{n-1}, i\right)$.

Lemma 2 gives index $(A)=i+1$ and it follows that

$$
\operatorname{dim}(A)= \begin{cases}\operatorname{dim}(B)=\operatorname{asc}\left(x^{\prime}\right)=\operatorname{asc}(x) & \text { if } i \leqslant x_{n-1}, \\ \operatorname{dim}(B)+1=\operatorname{asc}\left(x^{\prime}\right)+1=\operatorname{asc}(x) & \text { if } i>x_{n-1} .\end{cases}
$$

The result follows.

The inverse of this bijection is now straightforward. We omit the inductive proof.

Theorem 5. Let $A^{(1)}=(1) \in \operatorname{Int}_{1}$. Given $x=\left(x_{1}, x_{2}, \ldots, x_{n}\right) \in \mathcal{A}_{n}$, define the sequence of matrices $\left(A^{(2)}, \ldots, A^{(n)}\right)$ by $A^{(i+1)}=\phi\left(A^{(i)}, x_{i+1}\right)$ for $1 \leqslant i<n$. Then $\Gamma^{-1}(x)=A^{(n)}$. 


\section{$3 \quad$ Statistics and distributions}

In this section we show how statistics on the two structures are related under $\Gamma$. Many of the definitions concerning ascent sequences were stated in $[1, \S 5]$ and we recall them here.

Let $x=\left(x_{1}, \ldots, x_{n}\right)$ be a sequence of integers. For $k \leqslant n$, define $\operatorname{asc}_{k}(x)$ to be the number of ascents in the subsequence $\left(x_{1}, x_{2}, \ldots, x_{k}\right)$. If $x_{i}<x_{i+1}$, we say that $x_{i+1}$ is an ascent top.

Let $\operatorname{zeros}(x)$ be the number of zeros in $x$, and let last $(x):=x_{n}$. A right-to-left maximum of $x$ is an entry $x_{i}$ that has no larger entry to its right. We denote by $\operatorname{rmax}(x)$ the number of right-to-left maxima of $x$.

For sequences $x$ and $y$ of non-negative integers, let $x \oplus y=x y^{\prime}$, where $y^{\prime}$ is obtained from $y$ by adding $1+\max (x)$ to each of its letters, and juxtaposition denotes concatenation. For example $(3,2,0,1,2) \oplus(0,0,1)=(3,2,0,1,2,4,4,5)$. We say that a sequence $x$ has $k$ components if it is the sum of $k$, but not $k+1$, nonempty nonnegative sequences, and write $\operatorname{comp}(x)=k$.

Define $\mathfrak{a s c}(x)=\left\{i: i \in[n-1]\right.$ and $\left.x_{i}<x_{i+1}\right\}$. We denote by $\hat{x}$ the outcome of the following algorithm;

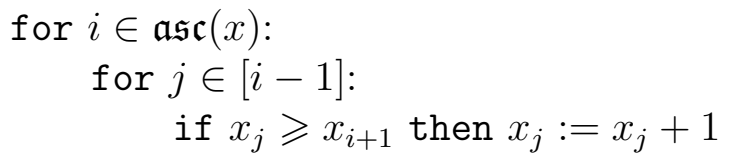

and call $\hat{x}$ the modified ascent sequence. For example, if $x=(0,1,0,1,3,1,1,2)$ then $\mathfrak{a s c}(x)=(1,3,4,7)$ and $\hat{x}=(0,3,0,1,4,1,1,2)$.

Note that the modified ascent sequence $\hat{x}$ has its ascents in the same positions as the original sequence, but that the ascent tops in $\hat{x}$ are all distinct. An ascent sequence $x$ is self-modified if $\hat{x}=x$.

Let $\operatorname{flip}(A)$ be the reflection of $A$ in its antidiagonal. Let blocks $(A)$ be the number of diagonal blocks in the matrix $A$.

Theorem 6. Let $A \in \operatorname{Int}_{n}$ and $x=\Gamma(A) \in \mathcal{A}_{n}$. Then

$$
\operatorname{rowsum}_{k}(A)=\left|\left\{j: \hat{x}_{j}=k-1\right\}\right| \text {. }
$$

Proof. By induction. The result is true for the single matrix $(1) \in \operatorname{lnt}_{1}$. Let us suppose that the result is true for all matrices $\operatorname{lnt}_{n-1}$ for some $n \geqslant 2$. Given $B \in \operatorname{Int}_{n-1}$, let $x=\left(x_{1}, \ldots, x_{n-1}\right)=\Gamma(B)$ and set $\hat{x}=\left(\hat{x}_{1}, \ldots, \hat{x}_{n-1}\right)$. Let $A=\phi(B, i)$ and $y=\left(x_{1}, \ldots, x_{n-1}, i\right)=\Gamma(A)$. Furthermore set $\hat{y}=\left(\hat{y}_{1}, \ldots, \hat{y}_{n}\right)$.

If index $(B) \leqslant i<\operatorname{dim}(B)$ then Add3 applies. In this case we find that $\operatorname{rowsum}_{k}(A)=$ $\operatorname{rowsum}_{k}(B)$ for all $0 \leqslant k \leqslant i, \operatorname{rowsum}_{i+1}(A)=1$, and $\operatorname{rowsum}_{k+1}(A)=\operatorname{rowsum}_{k}(B)$ for all $k \geqslant i+1$. Since $i>x_{n-1}$ we have that $n-1 \in \mathfrak{a s c}(x)$. This means that $\hat{y}$ is formed from $\hat{x}$ as follows: for all $1 \leqslant j \leqslant n-1$, if $\hat{x}_{j} \geqslant i$ then set $\hat{y}_{j}=\hat{x}_{j}+1$, and $\hat{y}_{n}=i$. By the 
induction hypothesis, for $k \leqslant i$ we have $\operatorname{rowsum}_{k}(A)=\operatorname{rowsum}_{k}(B)=\left|\left\{j: \hat{x}_{j}=k-1\right\}\right|=$ $\left|\left\{j: \hat{y}_{j}=k-1\right\}\right|$. Also, $\operatorname{rowsum}_{i+1}(A)=1=\left|\left\{j: \hat{y}_{j}=i\right\}\right|$ since $\hat{y}_{n}$ is the only entry that takes the value $i$. Finally for $k \geqslant i+1, \operatorname{rowsum}_{k+1}(A)=\operatorname{rowsum}_{k}(B)=\mid\left\{j: \hat{x}_{j}=\right.$ $k-1\}|=|\left\{j: \hat{y}_{j}=k\right\} \mid$.

The easier cases $i<\operatorname{index}(B)$ and $i=\operatorname{dim}(B)$ are dealt with in a similar manner so the proofs are omitted.

Given a square matrix $A$ and a sequence $x$, define the power series

$$
\begin{aligned}
\chi(x, q) & :=\sum_{i=1}^{|x|} q^{x_{i}}, & \bar{\chi}(x, q) & :=\sum_{x_{i} \text { rl-max }} q^{x_{i}}, \\
\lambda(A, q) & :=\sum_{i=1}^{\operatorname{dim}(A)} q^{\operatorname{rowsum}_{i}(A)}, & \bar{\lambda}(A, q) & :=\sum_{i=1}^{\operatorname{dim}(A)} A_{i, \operatorname{dim}(A)} q^{i-1} .
\end{aligned}
$$

Theorem 7. Suppose $A$ is the matrix corresponding to the ascent sequence $x$. Then

(i) $\operatorname{zeros}(x)=\operatorname{rowsum}_{1}(A)$;

(ii) $\operatorname{last}(x)=\operatorname{index}(A)-1$;

(iii) $\operatorname{asc}(x)=\operatorname{dim}(A)-1$;

(iv) $\operatorname{rmax}(\hat{x})=$ colsum $_{\operatorname{dim}(A)}(A)$;

(v) $\operatorname{comp}(\hat{x})=\operatorname{blocks}(A)$;

(vi) $\chi(\hat{x}, q)=\lambda(A, q)$;

(vii) $\bar{\chi}(\hat{x}, q)=\bar{\lambda}(A, q)$.

Proof. Most of the results follow from the sequence of rules applied to construct the matrix $A$ from the ascent sequence $x$.

(i) An entry $x_{j}=0$ if and only if the corresponding entry of the modified ascent sequence $\hat{x}_{j}=0$. This result now follows from Theorem 6 with $i=1$.

(ii) and (iii) follow directly from Theorem 4.

(iv) is an immediate consequence of the proof of (vii) below with $q=1$.

(v) We now show that $\operatorname{comp}(\hat{x})=\operatorname{blocks}(A)$. It suffices to prove that $\hat{x}=\hat{y} \oplus \hat{z}$ with $|y|=\ell$ and $|z|=m$ iff $A=\left(\begin{array}{cc}A_{y} & 0 \\ 0 & A_{z}\end{array}\right)$ with $A_{y} \in \operatorname{lnt}_{\ell}$ and $A_{z} \in \operatorname{Int}_{m}$, where $\Gamma\left(A_{y}\right)=y$ and $\Gamma\left(A_{z}\right)=z$. 
Let us assume that $\hat{x}=\hat{y} \oplus \hat{z}$. The first $\ell$ steps of the construction of $A$ give $A_{y}$ where $\operatorname{dim}\left(A_{y}\right)=\operatorname{asc}(y)+1$. Next, since $\hat{x}_{\ell+1}=1+\max \left\{\hat{x}_{j}: j \leqslant \ell\right\}$, the addition rule Add2 is used, and we have

$$
A^{\prime}=\left(\begin{array}{cc}
A_{y} & 0 \\
0 & 1
\end{array}\right)
$$

where the new 1 is in position $(\operatorname{asc}(y)+2, \operatorname{asc}(y)+2)$. All subsequent additions, $x_{j}$ for $\ell+1<j \leqslant \ell+m$ are such that $\hat{x}_{j} \geqslant 1+\operatorname{asc}(y)$, and so do not affect the first $\operatorname{asc}(y)+1$ rows or columns of $A^{\prime}$. Further to this, the construction that takes place for steps $\ell+1, \ldots, \ell+m$ has the same relative order as the construction of $A_{z}$. This gives

$$
A=\left(\begin{array}{cc}
A_{y} & 0 \\
0 & A_{z}
\end{array}\right) \text {. }
$$

Conversely assume that $A=\left(\begin{array}{cc}B & 0 \\ 0 & C\end{array}\right)$ with $B \in \operatorname{Int}_{\ell}$ and $C \in \operatorname{Int}_{m}$ and $n=\ell+m$. The first $m$ removal operations only affect entries in $C$ since there is at least one nonzero entry in every row and column of $C$. Thus $\widehat{x}_{\ell+1}, \ldots, \widehat{x}_{n} \geqslant \operatorname{dim}(B)$ and in particular, $\widehat{x}_{\ell+1}=\operatorname{dim}(B)$. Note that the sequence $\left(x_{\ell+1}-\operatorname{dim}(B), \ldots, x_{n}-\operatorname{dim}(B)\right)=\left(z_{1}, \ldots, z_{m}\right)$ is an ascent sequence which is $\Gamma(C)$. After these removals, we are left with the matrix $B$, and since it is in $\operatorname{Int}_{\ell}$, the values $x_{1}, \ldots, x_{\ell}<\operatorname{dim}(B)$. Let $y_{j}=x_{j}$ for all $j \leqslant \ell$. Consequently one has $\hat{x}=\hat{y} \oplus \hat{z}$.

(vi) is an immediate consequence of Theorem 6 .

Finally, part (vii) is proved by induction as follows. The result is clearly true for the single matrix $(1) \in \operatorname{Int}_{1}$. Assume it is true for all matrices in $\operatorname{Int}_{n-1}$ for some $n \geqslant 2$. Let $B \in \operatorname{Int}_{n-1}$ with $x^{\prime}=\left(x_{1}, \ldots, x_{n-1}\right)=\Gamma(B)$. Let $A=\phi(B, i)$ with $x=\left(x_{1}, \ldots, x_{n}\right)=$ $\Gamma(A)$. Then

$$
\bar{\lambda}(A, q)= \begin{cases}\bar{\lambda}(B, q)+q^{i} & \text { if } i \leqslant \operatorname{index}(B)-1 \\ q^{i}+\sum_{j=i+1}^{\operatorname{dim}(B)} B_{j, \operatorname{dim}(B)} q^{j} & \text { otherwise. }\end{cases}
$$

Similarly,

$$
\bar{\chi}(\hat{x}, q)= \begin{cases}\bar{\chi}\left(\widehat{x^{\prime}}, q\right)+q^{i} & \text { if } i \leqslant x_{n-1} \\ q^{i}+\sum_{\operatorname{rl}-\max \widehat{x_{j}^{\prime}} \geqslant i} q^{\widehat{x_{j}^{\prime}}+1} & \text { otherwise. }\end{cases}
$$

From the induction hypothesis, for the case $i \leqslant \operatorname{index}(B)-1=x_{n-1}$, we have $\bar{\lambda}(B, q)=$ $\bar{\chi}\left(\widehat{x^{\prime}}, q\right)$. Otherwise,

$$
\sum_{j=i+1}^{\operatorname{dim}(B)} B_{j, \operatorname{dim}(B)} q^{j}=\sum_{\text {rl-max } \widehat{x_{j}^{\prime}} \geqslant i} q^{\widehat{x_{j}^{\prime}}+1}
$$


since these power series are simply $\bar{\lambda}(B, q)$ and $\bar{\chi}\left(\widehat{x^{\prime}}, q\right)$, respectively, without the first $i$ powers of $q$. Thus $\bar{\chi}(\hat{x}, q)=\bar{\lambda}(A, q)$.

The above results, used in conjunction with the flip operation, allow us to prove the following equidistribution result on ascent sequences.

Theorem 8. For all $n \geqslant 1$, zeros $(\cdot)$ and $\operatorname{rmax}(\hat{\cdot})$ are equidistributed on the set $\mathcal{A}_{n}$.

Proof. The operation flip shows that $\operatorname{rowsum}_{1}(\cdot)$ and $\operatorname{colsum}_{\operatorname{dim}(\cdot)}(\cdot)$ are equidistributed on $\operatorname{Int}_{n}$. Apply Theorem 7 (i) and (iv) to find that $\operatorname{zeros}(\cdot)$ and $\operatorname{rmax}(\hat{\cdot})$ are equidistributed on $\mathcal{A}_{n}$.

In dealing with compositions of an integer, the number of parts in a composition is a natural statistic by which the collection of compositions may be refined. The next theorem gives the relation between the number of non-zero parts in our 'matrix composition of the integer $n$ ' and the ascent sequence to which it corresponds.

Theorem 9. Let $x=\Gamma(A)$ where $A \in \operatorname{lnt}_{n}$. The number of positive entries in $A$ is equal to $n$ less the number of equal adjacent entries in $x$.

Proof. Suppose that $x=\left(x_{1}, \ldots, x_{n}\right)$. Let $A^{(i)}$ be the matrix corresponding to $\left(x_{1}, \ldots, x_{i}\right)$ and define $N_{i}$ to be the number of positive entries in $A^{(i)}$. Since $A^{(1)}=(1)$ we have $N_{1}=1$. Given $i \geqslant 2$, if $x_{i}<x_{i-1}$ then one of the zeros in $A^{(i-1)}$ becomes a one in $A^{(i)}$ so that $N_{i}=N_{i-1}+1$. If $x_{i}=x_{i-1}$ then value $\left(A^{(i-1)}\right)$ is increased by one to give $A^{(i)}$, so in this case $N_{i}=N_{i-1}$. Otherwise $x_{i}>x_{i-1}$ and a new row and column is inserted into $A^{(i-1)}$ to give $A^{(i)}$, and a 1 is introduced, giving $N_{i}=N_{i-1}+1$. These equalities may be summarized by $N_{i}=N_{i-1}+a_{i}$ where $a_{i}=\mathbf{1}\left(x_{i} \neq x_{i-1}\right)$. So the number of positive entries in $A$ is

$$
1+a_{2}+\ldots+a_{n}=1+(n-1)-\sum_{i} \mathbf{1}\left(x_{i}=x_{i-1}\right)
$$

which is $n$ less the number of equal adjacent entries in $x$.

Theorem 10. The trace $\operatorname{tr}(A)$ is equal to the number of entries $x_{i}$ in the corresponding sequence $x$ such that $x_{i}=\operatorname{asc}_{i}(x)$.

Proof. First note that if $i>1$, then $x_{i}=\operatorname{asc}_{i}(x)$ if either $x_{i}=1+\operatorname{asc}_{i-1}(x)$ or if $x_{i}=x_{i-1}=\cdots=x_{i-j}=1+\operatorname{asc}_{i-j-1}(x)$ for some $j \geqslant 1$.

Now consider the step-by-step process of building $A$. If $x_{i}=1+\operatorname{asc}_{i-1}(x)$, then the matrix dimension increases, and a new entry 1 is inserted at the end of the diagonal. If $j>0$, and $x_{i+j}=\cdots=x_{i}=1+\operatorname{asc}_{i-1}(x)$, then the same entry gets increased by one.

Entries at the diagonal can never decrease, and the two cases above are the only times an entry on the diagonal can increase, so the result follows. 
Define a run in the sequence $x=\left(x_{1}, \ldots, x_{n}\right)$ to be a maximal subsequence of adjacent equal elements, that is, a subsequence $\left(x_{i}, x_{i+1}, \ldots, x_{i+j}\right)$ such that $x_{i}=x_{i+1}=\cdots=x_{i+j}$, where $x_{i-1} \neq x_{i}$ if $i>1$, and $x_{i+j} \neq x_{i+j+1}$ if $i+j<n$. If $x_{i}=y$, we say the run is a $y$-run.

Theorem 11. Let $A \in \operatorname{Int}_{n}$, and suppose that $x=\Gamma(A)$ is the corresponding sequence. The following three equalities hold.

(i) $A_{1,1}$ equals the length of the starting 0-run.

(ii) $\operatorname{value}(A)$ equals the length of the ending $x_{n}$-run.

(iii) $A_{\operatorname{dim}(A), \operatorname{dim}(A)}$ equals the length of the last $y$-run whose first entry $x_{i}=y$ satisfies $x_{i}=1+\operatorname{asc}_{i-1}(x)$.

Furthermore, the distribution of all three statistics on matrices are the same, as is the distribution of all three statistics on ascent sequences.

Proof. Using the standard method of building the matrix according to the ascent sequence it is straightforward to check that the three equalities hold.

To show that the first two statistics on ascent sequences are equidistributed, a simple bijection can be used. Assume that $x$ is of the form $\left(0^{a}, y, i^{b}\right)$, where the subsequence $y$ starts with 1 , and does not end with $i$. Map $x$ to $\tilde{x}=\left(0^{b}, y, i^{a}\right)$. It is obvious that this is a bijection (and also an involution), and that the result follows.

The third statistic also have the same distribution by symmetry — it is equal to flip $(A)_{1,1}$.

Remark 12. The observant reader may have noticed that there is a fourth pair missing from the above theorem: the last positive entry in the first row of the matrix, and its counterpart for sequences. The counterpart is the length of a subsequence of zeros, but the rule for deciding which is quite complicated.

Conjecture 13. For ascent sequences $x$, the distribution of $\operatorname{zeros}(x)$, or equivalently, the distribution of $\operatorname{rmax}(\hat{x})$, is the same as the distribution of the length of the first strictly increasing subsequence of $x$.

\section{Binary, positive diagonal, and bidiagonal matrices}

We now turn to some natural subclasses of matrices. These are binary matrices, matrices that have no zeros on their diagonal, and bidiagonal matrices.

First, let us note that it is easy to see that the collection of diagonal matrices in $\operatorname{lnt}_{n}$ correspond to compositions of the integer $n$. Given such a matrix $A=\operatorname{diag}\left(a_{0}, \ldots, a_{k}\right) \in$ 
$\operatorname{Int}_{n}$, the corresponding ascent sequence is

$$
\Gamma(A)=\left(0^{a_{0}}, 1^{a_{1}}, \ldots, k^{a_{k}}\right) \text {, with } a_{0}+a_{1}+\cdots+a_{k}=n .
$$

It is known that the binary matrices in $\operatorname{lnt}_{n}$ correspond to interval orders with no repeated holdings [3]. These are a subclass of interval orders, which were shown in [1] to be in bijection with ascent sequences. From Theorem 9 we have the following two results:

Corollary 14. A matrix $A \in \mathrm{Int}_{n}$ is binary if and only if the corresponding ascent sequence $x=\Gamma(A)$ contains no two equal consecutive entries.

Corollary 15. Let $A \in \operatorname{Int}_{n}$ be the matrix corresponding to the ascent sequence $x$. Then the sum $\sum_{i, j} \max \left\{0,\left(A_{i, j}-1\right)\right\}$ equals the number of pairs $\left(x_{i}, x_{i+1}\right)$ in $x$ such that $x_{i}=$ $x_{i+1}$.

Next we classify those matrices in $\operatorname{Int}_{n}$ that have only positive diagonal entries. Let us point out that the following class of ascent sequences correspond to permutations that avoid the pattern $3 \overline{1} 52 \overline{4}$, see [1, Prop. 9].

Theorem 16. The matrix $A=\Gamma^{-1}(x)$ has only positive entries on the diagonal exactly when the sequence $x$ is self-modified, that is when $x=\hat{x}$.

Proof. Consider the sequence of addition rules used to build $A$. If $A$ has no zeros on the diagonal, it means that Add3 was never used.

This means that for the sequence $x$, for all $i, x_{i-1} \geqslant x_{i}$ or $x_{i}=1+\operatorname{asc}_{i-1}(x)$. In other words all ascents are maximal. This is exactly the condition for a sequence to be selfmodified: a sequence is not self-modified if and only if there exist $i$ and $j<i$ such that $x_{j} \geqslant x_{i+1}$ and $x_{i}<x_{i+1}$.

\subsection{Bidiagonal matrices and order-consecutive set partitions}

Consider the subclass $\mathrm{Bi}_{n} \subseteq$ Int $_{n}$ of matrices defined to be the bidiagonal matrices in Int ${ }_{n}$. It turns out that there is a natural bijection between $k \times k$ matrices in $\mathrm{Bi}_{n}$ and so called order-consecutive set partitions, [4], of $[n]$ into $k$ parts. A set partition is order-consecutive if the parts $P_{1}, P_{2}, \ldots, P_{k}$ can be ordered as

$$
P_{\pi_{1}}, P_{\pi_{2}}, \ldots, P_{\pi_{k}}
$$

such that each set $\bigcup_{i=1}^{j} P_{\pi_{i}}$ is an interval in $[n]$. For example,

$$
\{\{1,2,3\},\{4,9\},\{5\},\{6,7\},\{8\}\}
$$

is order-consecutive, for we can order the parts as

$$
\{5\},\{6,7\},\{8\},\{4,9\},\{1,2,3\} \text {. }
$$


The set partition $\{\{1,3\},\{2,4\}\}$ however is not order-consecutive.

An order-consecutive set partition of $[n]$ into $k$ parts can be represented as the sequence 1 to $n$, with $k$ pairs of parenthesis inserted (see [4]). For example,

$$
\{\{1,2,3\},\{4,9\},\{5\},\{6,7\},\{8\}\}
$$

is represented as $(123)(4(5)(67)(8) 9)$. Note that each pair of parenthesis are placed as close together as possible. Thus, $(1(2))$ is not a valid representation - the proper one for this partition is $(1)(2)$. These representations for order-consecutive partitions obey an additional constraint [4, Lemma 5]:

Constraint *: If all )(-pairs are deleted, the remaining pairs are completely nested, i.e. removing the numbers we are left with $((\cdots()) \cdots)$.

Given an order-consecutive set partition $P=\left(P_{1}, \ldots, P_{k}\right)$ of $[n]$, let us write $\alpha(P)$ for this representation involving parentheses. We form a bidiagonal matrix $B=B(P)$ as follows. Let $B(P)$ initially be the $k \times k$ matrix with all elements zero except a one at the top left corner. Read the sequence $\alpha(P)$ from left to right, starting with 1 . When reading the sequence, if the next symbol is a number, increase the element in the current position of $B(P)$ by one. If it is a parenthesis increase either the row index or column index of $B(P)$ by one, whichever allows us to stay on the diagonal and bidiagonal.

For example, the partition above with $\alpha(P)=(123)(4(5)(67)(8) 9)$ gives the matrix

$$
B(P)=\left(\begin{array}{ccccc}
3 & 0 & 0 & 0 & 0 \\
0 & 1 & 1 & 0 & 0 \\
0 & 0 & 0 & 2 & 0 \\
0 & 0 & 0 & 0 & 1 \\
0 & 0 & 0 & 0 & 1
\end{array}\right)
$$

Theorem 17. There is a one-to-one correspondence between $k \times k$ matrices in $\mathrm{Bi}_{n}$ and order-consecutive set partitions of $[n]$ into $k$ parts.

Proof. It is clear from above construction that if $P$ is an order-consecutive set partition, then $B(P) \in \mathrm{Bi}_{n}$. We show it is one-to-one by defining the inverse. The numbers 1 to $n$ are to be written down in order, with parenthesis interspersed. Start by writing (. Next visit the elements in the matrix in order $(1,1),(1,2),(2,2),(2,3), \ldots$ If the number $m$ is encountered, write down the next $m$ numbers and then a $\mid$. End with a ).

Now change each $\|$ into $)($. Note that there can be no more than two consecutive |'s.

To finish, we need to change each remaining | into either ) or (. However, using constraint $*$, there is a unique way of doing this.

Corollary 18 ([4]). The number of $k \times k$ bidiagonal matrices in $\operatorname{lnt}_{n}$ is

$$
\sum_{j=0}^{k-1}\left(\begin{array}{c}
n-1 \\
2 k-j-2
\end{array}\right)\left(\begin{array}{c}
2 k-j-2 \\
j
\end{array}\right) .
$$


Furthermore, from the construction above one may notice that the $j+1$ th term in the sum counts the number of matrices with exactly $j$ zeros in the diagonal and bidiagonal.

Theorem 19. The set of ascent sequences $x$ such that $x=\Gamma(A)$ for $A \in \mathrm{Bi}_{n}$ are those sequences $x=\left(x_{1}, \ldots, x_{n}\right)$ which satisfy

$$
x_{i} \geqslant \operatorname{asc}_{i}(x)-1
$$

for $1 \leqslant i \leqslant n$.

Proof. Induction on $n$. The $n=1$ case is trivial, so assume that $A \in \operatorname{lnt}_{n}$ is bidiagonal, and that $x=\left(x_{1}, x_{2}, \ldots, x_{n}\right)=\Gamma(A)$ obeys $(3)$.

Consider two cases. First assume the last column of $A$ ends with $(0, a)$ for some $a \geqslant 1$. This means that $x$ ends with $x_{n+1-a}=x_{n+2-a}=\cdots=x_{n}=\operatorname{asc}_{n+1-a}(x)=\operatorname{asc}_{n}(x)$.

Let $y=\left(x_{1}, \ldots, x_{n}, x_{n+1}\right)$ and $B=\Gamma^{-1}(y)$. Consider the three subcases $x_{n}<x_{n+1}$, $x_{n}=x_{n+1}$ and $x_{n}>x_{n+1}$.

If $x_{n+1}=x_{n}+1$ then $B=\Gamma^{-1}(y)$ is $\left(\begin{array}{cc}A & 0 \\ 0 & 1\end{array}\right)$, and bidiagonal by the induction hypothesis. Also, $x_{n+1}=\operatorname{asc}_{n}(y)+1=\operatorname{asc}_{n+1}(y)$, so $x_{n+1} \geqslant \operatorname{asc}_{n+1}(y)-1$.

If $x_{n+1}=x_{n}$ then $B$ is $A$ with the entry at position $(\operatorname{dim}(A), \operatorname{dim}(A))$ increased by one, and again bidiagonal. Furthermore, $x_{n+1}=\operatorname{asc}_{n+1}(y) \geqslant \operatorname{asc}_{n+1}(y)-1$.

If $x_{n+1}=x_{n}-m$ for $m>0$, then $B$ is $A$ with the 0 at position $(\operatorname{dim}(A)-m, \operatorname{dim}(A))$ increased to a 1 , and $A$ is bidiagonal if and only if $m=1$. Also, $x_{n+1}=\operatorname{asc}_{n}(x)-m$, so $x_{n+1} \geqslant \operatorname{asc}_{n+1}(y)-1$ only for $m=1$.

This proves the theorem in first case. The second case, when the last column of $A^{\prime}$ ends with $(a>0, b>0)$ is handled in a similar way.

\section{$5 \quad$ Some challenging questions}

We end this paper with two challenging questions.

Question 20. If $x=\Gamma(A)$ for some $A \in \operatorname{Int}_{n}$, then what is the sequence $y=y(x)$ for which $y=\Gamma($ flip $(A))$ ?

In terms of $(\mathbf{2}+\mathbf{2})$-free posets, this question is equivalent to asking for the ascent sequence $y$ that corresponds to the dual poset $P^{\star}$, where the poset $P$ is generated by the ascent sequence $x$.

Adding two upper triangular matrices of the same dimension yields another upper triangular matrix of the same dimension.

Question 21. Adding two matrices of the same size is a commutative mapping Int $_{n} \times$ $\mathrm{Int}_{m} \mapsto \mathrm{Int}_{n+m}$. How does this operation act on the corresponding ascent sequences? Furthermore, how does this addition operation act on the corresponding posets? 


\section{References}

[1] M. Bousquet-Mélou, A. Claesson, M. Dukes and S. Kitaev, $(\mathbf{2}+\mathbf{2})$-free posets, ascent sequences and pattern avoiding permutations, Journal of Combinatorial Theory, Series A, to appear, arXiv:0806.0666.

[2] M. Dukes, S. Kitaev, J. Remmel and E. Steingrímsson, Enumerating $(\mathbf{2}+\mathbf{2})$-free posets by indistinguishable elements, preprint 2010.

[3] P. C. Fishbrun, Interval Graphs and Interval Orders, Wiley, New York, 1985.

[4] F. K. Hwang and C. L. Mallows, Enumerating Nested and Consecutive Partitions, Journal of Combinatorial Theory, Series A 70 (1995), 323-333.

[5] J. Mitas, Tackling the jump number of interval orders, Order 8 (1991), 115-132. 